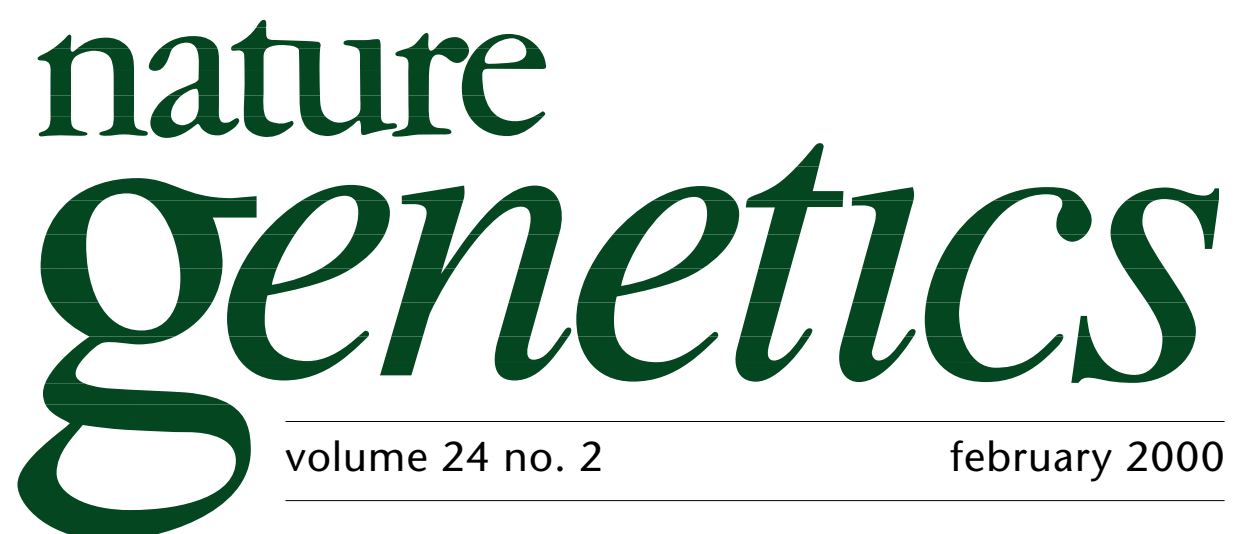

\title{
Census, race and science
}

Jesus Christ was born in Bethlehem because his parents had to register there with the Roman authorities, who sought information on the population of their Empire. Roughly two millennia later, the United States government will ask its citizens to take part in a similar endeavour, the Census 2000. The US Constitution calls for a count of the nation's populace every ten years to apportion representation in the House of Representatives. Census data provide the basis for domestic policy and are used to monitor and enforce compliance with civil-rights statutes and anti-discrimination laws. As part of this 'snapshot' of the nation, individuals will be asked about their race and ethnicity. These terms mean different things to different people (see box for selected dictionary definitions). The Census Bureau does not offer its own definitions, but makes it explicit that the individual is the only one who should classify her- or himself.

In the past, five racial categories were provided: American

"For those of a colonial mindset, this swirling postcolonial world is blurring distinctions and challenging received wisdoms at an alarming rate. It is uncomfortably clear to those of the 'old school' that the hitherto familiar cultural signifiers of belonging are no longer the preserve of one group to the exclusion of another. . . However, it is not only those of a colonial mindset who must open themselves to change."

-Caryl Phillips, The Guardian newspaper.
Indian or Alaska Native, Asian, Black or African American, Native Hawaiian or Other Pacific Islander, and White. In addition, people were asked about their ethnicity, that is, whether they were of Hispanic (or Latino) origin. Only one race could be selected, and people also had to state whether they were 'Hispanic or Latino', or not. In this year's census, a sixth racial category called 'Other Race' will be added, and, for the first time, a person can select multiple races (leading to 63 possible permutations). It is not yet clear how the Census will tabulate this information; hopefully social and life scientists will have access to the detailed numbers and percentages, even if these are not deemed useful for all administrative purposes. The increasing diversity of the nation's population is the reason cited by the US Office of Management and Budget (OMB) for these changes. It will be interesting to see whether this will be reflected in the results and how people categorize themselves.

That race in this context is not a scientific term is generally agreed upon by scientists - and a message that cannot be repeated often enough. Whether racial classification is useful at all is debatable. In 1997, the American Association of Anthropologists (AAA) urged the US government to phase out their use of the term 'race' in the collection of federal data, because the concept of race is a social and cultural construction with no scientific justification in human biology. Instead of race, they suggest the use of ethnic categories that better reflect the diversity of the US population. The AAA recommended that the OMB combine the separate 
race and ethnicity categories into one question for the 2000 Census, and eliminate 'race' as a category by the time planning begins for the 2010 Census. The AAA statement also calls for research to determine what terms best capture human variability in ways understood by the American people, and argues that "probably the clearest data on human variation come from genetic studies. Genetic data do show differences between groups, and these can potentially trace an individual's likely geographic origin. Nevertheless, these data also show that any two individuals within a particular population are as different genetically as any two people selected from any two populations in the world."

Census 2000, in which US residents will be asked to state their race and ethnicity, provides an ideal opportunity for geneticists to reiterate that their research does not support the concept of race as a biological entity.

\section{A diversity of definitions}

\section{Race}

- A vague, unscientific term for a group of genetically related people who share certain physical characteristics

- A distinct ethnic group characterized by traits that are transmitted through their offspring

- Each of the major divisions of humankind, having distinct physical characteristics

- A group of individuals who are more or less isolated geographically or culturally, who share a common gene pool, and whose allele frequencies at some loci differ from those of other populations

\section{Ethnic group}

- A population of individuals organized around an assumption of common cultural origin

- Individuals with a common national or cultural tradition

- A social group or category of the population that, in a larger society, is set apart and bound together by common ties of race, language, nationality or culture At present, the messages are mixed: on one hand, the public is told that there is no scientific basis for race, and that there is more variation within populations than between populations. On the other, scientists use racial terms when describing research results, such as an increased risk for breast cancer in Jews or for prostate cancer in Blacks, and frequently emphasize population-specific markers, alleles and disease susceptibility. This is a complex and sensitive topic, but we must become better in educating the public about the scientific questions that are being addressed, and what we learn about human diversity.

At the same time, we should look closer at the issues of race, populations and ethnicity in scientific discourse, which is often associated with poorly defined lay terminology. This is not just a matter of sloppy language, but reflects the imprecise use of racial and ethnic classifications in biomedical research. Throughout history scientists have used social and politically determined racial categories to make scientific comparisons between races - with little or no discussion about the meaning or rationale. The US National Institutes of Health Revitalization Act of 1993 calls for clinical trials to enroll minority group members based on traditionally defined racial and ethnic groups (and on an individual's self-selection into those groups), and the documentation of results by race. Race might be a proxy for discriminatory experiences, diet or other environmental factors. If these factors are relevant to the question asked, race can be a valid variable in scientific studies. There is no justification, however, to use race as a substitute for other parameters that can be measured, such as genetic variation or differences in metabolism. Increasing mobility and admixture will also make it less and less likely that the substitution will yield reasonable results. Instead, available genotyping methods will make it possible to define genetic subgroups (which do not correlate with skin colour, language or religion) that are relevant to a particular scientific question.

The laudable objective to find means to improve the health conditions for all or for specific populations must not be compromised by the use of race or ethnicity as pseudo-biological variables. From now on, Nature Genetics will therefore require that authors explain why they make use of particular ethnic groups or populations, and how classification was achieved. We will ask reviewers to consider these parameters when judging the merits of a manuscriptwe hope that this will raise awareness and inspire more rigorous design of genetic and epidemiological studies.

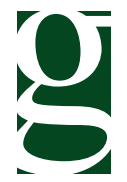

